\title{
Vítimas de maus-tratos, negligência ou abandono em estado do Nordeste Brasileiro
}

RESUMO | Objetivo: descrever a situação epidemiológica de pessoas que foram vítimas de maus-tratos, negligência ou abandono em um estado do nordeste brasileiro. Método: Trata-se de estudo exploratório e descritivo, com abordagem quantitativa e dados retrospectivos, desenvolvido com dados obtidos do Sistema de Informação de Agravos de Notificação. Resultados: A população compreendeu pessoas atendidas e notificadas nos serviços próprios e conveniados ao Sistema Único de Saúde, por causas de maus-tratos, negligência ou abandono, entre 2015 e 2018. Observou-se que a maior parte era do sexo feminino (59,6\%), na faixa etária de até 9 anos $(56,4 \%)$ e residentes da zona urbana $(79,1 \%)$. Quanto as pessoas que cometeram maus-tratos, negligência ou abandono, a maioria era do sexo feminino, não havia ingerido bebida alcóolica e a violência foi motivada por conflito geracional (20\%). Conclusão: Evidenciou-se a necessidade de políticas públicas que proteja as crianças e as pessoas que estão em situação de vulnerabilidade.

Palavras-chaves: Violência; Negligência; Maus-tratos Infantis; Epidemiologia.

\begin{abstract}
Objective: describes an epidemiological situation of people who have been victims of abuse, neglect or abandonment in a state in northeastern Brazil. Method: This is an exploratory and descriptive study, with a quantitative approach and retrospective data, developed with data obtained from the Notifiable Diseases Information System. Results: The population comprised people attended and notified in the services themselves and under the Unified Health System, due to maltreatment, neglect or abandonment, between 2015 and 2018. It was observed that the majority were female (59, 6\%), aged up to 9 years (56.4\%) and residents of the urban area (79.1\%). As for people who committed abuse, neglect or abandonment, the majority were female, had not drunk alcohol and violence was motivated by generational conflict (20\%). Conclusion: The need for public policies to protect children and people who are in a situation of vulnerability was highlighted.
\end{abstract}

Keywords: Violence; Negligence; Child abuse; Epidemiology.

RESUMEN | Objetivo: describe una situación epidemiológica de personas que han sido víctimas de abuso, negligencia o abandono en un estado del noreste de Brasil. Método: Se trata de un estudio exploratorio y descriptivo, con enfoque cuantitativo y datos retrospectivos, desarrollado con datos obtenidos del Sistema de Información de Enfermedades Notificables. Resultados: La población estuvo conformada por personas atendidas y notificadas en los propios servicios y en el Sistema Único de Salud, por maltrato, negligencia o abandono, entre 2015 y 2018. Se observó que la mayoría eran mujeres (59, 6\%), de hasta 9 años $(56,4 \%)$ y residentes del casco urbano $(79,1 \%)$. En cuanto a las personas que cometieron maltrato, abandono o abandono, la mayoría eran mujeres, no habían bebido alcohol y la violencia estuvo motivada por conflicto generacional (20\%). Conclusión: Se destacó la necesidad de políticas públicas para proteger a la niñez y a las personas en situación de vulnerabilidad.

Palabras claves: Violencia; Negligencia; Abuso infantil; Epidemiología.

\section{Aldenira Joacla Caetano da Silva}

Enfermeira. Universidade Federal do Rio Grande do Norte. Especialista em Ginecologia e Obstetrícia, Especialista em Urgência e Emergência, Especialista em Auditoria nos Serviços de Saúde.

ORCID: 0000-0002-4815-2930

\section{Emanoele Belchior de Medeiros}

Enfermeira. Universidade Federal do Rio Grande do Norte. Especialista em Ginecologia e Obstetrícia, Especialista em Urgência e Emergência.

ORCID: 0000-0002-2625-6798

\section{Illana Cecylia Silva Basílio}

Enfermeira, Centro Universitário Facex (UNIFACEX).

ORCID: 0000-0002-0000-4170

Recebido em: 23/11/2020

Aprovado em: 01/12/2020

\section{Janaina Kelly Alves Barbosa}

Enfermeira. Universidade Federal do Rio Grande do Norte. Especialista em Ginecologia e Obstetrícia, Especialista em Gestão do Trabalho e da Educação na Saúde. ORCID: 0000-0001-8072-7055

\section{Robson Egidio da Silva}

Enfermeiro. Faculdade Mauricio de Nassau. Especialista em UTI.

ORCID: 0000-0003-3665-0761

\section{INTRODUÇÃO}

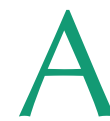
partir da década de 1980, o tema da violência passou a ocupar lugar de destaque nas agendas de debates políticos e sociais e no setor saúde, passando a ser incorporado como questão de saúde pública e desafio para os gestores do Sistema Único de Saúde (SUS). ${ }^{1}$
A Organização Mundial de Saúde (OMS) apresentou em seu Relatório Mundial sobre violência e saúde que todo ano, mais de um milhão de pessoas perdem a vida e muitas outras sofrem lesões não fatais por causas violentas. O contexto Brasileiro não foge da realidade mundial e por ser um país subdesenvolvido os dados tendem a ser mais expressivos. No Brasil, somente na década de 90, morreram mais de um milhão de pessoas por violências e acidentes e dessas, cerca de 400 mil faleceram por homicídios. ${ }^{1}$

Como falar acerca da violência e não citar os crescentes casos e relatos de maus-tratos, negligências e abandono por parte de pessoas tidas como responsáveis legais e ou entes familiares.

A violência é definida como qualquer atitude ou comportamento que usa da for- 
ça e do poder para causar intencionalmente dano ou intimidação contra a integridade física ou psicológica, através de autoagressões, agressões interpessoais ou coletivas. ${ }^{2}$

Salienta-se que as violências passaram a integrar a lista de notificação compulsória a partir de 2011, com a publicação da Portaria $n^{\circ} 104$, de 25 de janeiro de 2011, e posteriormente por meio da Portaria $\mathrm{n}^{\circ} 1.271$, de 6 de junho de 2014, universalizando esse procedimento para todos os serviços de saúde. A ficha de notificação individual deve ser utilizada para registrar qualquer caso suspeito ou confirmado de violência doméstica/intrafamiliar, sexual, autoprovocada, tráfico de pessoas, trabalho escravo, trabalho infantil, tortura, intervenção legal e violências homofóbicas contra as mulheres e os homens em todas as idades. ${ }^{3}$

No entanto, mesmo diante de um cenário tão alarmante, ainda existem subnotificação dos dados e muitos profissionais desconhecem ou não reconhecem a importância da notificação dos dados por meio de um instrumento de coleta. Desse modo, torna-se importante a capacitação dos profissionais de saúde para a melhoria da qualidade das informações, tendo em vista o diagnóstico real da situação, para que sejam realizadas as ações de vigilância epidemiológica e de prevenção da violência. ${ }^{4}$

Portanto, a notificação é uma obrigação institucional, cabendo aos serviços, aos gestores e aos profissionais da saúde a responsabilidade de realizar a notificação compulsória em conformidade com a legislação vigente, pois a ficha de notificação/investigação coleta e fornece dados de suma importância para elaboração de estratégias e planos de ação que visam combater a violência.

Outro avanço em relação à prevenção das violências foi a implantação do Sistema de Vigilância de Violências e Acidentes (VIVA), entre os anos de 2006 a 2008, nos serviços de referência para violências, tais como centros de referência para violências, centros de referência para Infecções Sexualmente Transmissíveis (IST) e Síndrome da Imunodeficiência Adquirida (SIDA), ambulatórios especializados e maternidades. E a partir de 2009, o VIVA passou a integrar o Sistema de Informação de Agravos de Notificação (SINAN), associando-se a lista de notificação compulsória em unidades sentinela. ${ }^{5}$

Em conformidade com o que foi exposto, considera-se os maus-tratos, negligência e abandono como temáticas de extrema relevância para a sociedade, por se tratar de problemas de saúde pública no qual esfacela e provocam danos muitas vezes irreparáveis, tanto para o indivíduo quanto para os demais membros das famílias. No entanto, esse problema pode ser evitável e seu enfrentamento demanda esforços coordenados e sistematizados de diversos setores governamentais, segmentos sociais e da população em geral, portanto a busca por uma solução deve ser exaustivamente pesquisada até que este não seja mais um causador de vítimas.

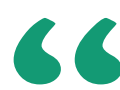

Nesse contexto que se insere o presente artigo, que objetiva descrever a situação epidemiológica de pessoas que foram vítimasde maustratos, negligência ou abandono em um estado do nordeste brasileiro.

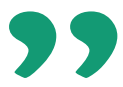

Nesse contexto que se insere o presente artigo, que objetiva descrever a situação epidemiológica de pessoas que foram vítimasde maus-tratos, negligência ou abandono em um estado do nordeste brasileiro.
MÉTODO

Trata-se de estudo exploratório e descritivo, com abordagem quantitativa e dados retrospectivos. Os dados foram obtidos do Sistema de Informação de Agravos de Notificação (SINAN) - Fichas de Notificação de Violência Doméstica/Intrafamiliar registrada e Ficha de Violências e Acidentes em Serviços Sentinela de Urgência e Emergência, disponibilizados pela Secretaria de Vigilância em Saúde da Secretaria Estadual e Municipal de Saúde por meio do portal eletrônico do Departamento de Informática do Sistema Único de Saúde (DATASUS).

A população compreendeu todas as pessoas que foram atendidas e notificadas nos serviços próprios e conveniados ao SUS, por causas de maus-tratos, negligência ou abandono e entre 2015 e 2018 em Natal, Capital do Rio Grande do Norte.

De acordo com ficha de notificação a definição de Violência Interpessoal/ Autoprovocada diz respeito a um "caso suspeito ou confirmado de violência doméstica/intrafamiliar, sexual, autoprovocada, tráfico de pessoas, trabalho escravo, trabalho infantil, tortura, intervenção legal e violências homofóbicas contra mulheres e homens em todas as idades".

Para que a pesquisa ocorresse foram adotados os seguintes procedimentos: encaminhamento de ofício ao Secretário de Estado da Saúde Pública do Rio grande do Norte, no qual informou sobre a pesquisa e solicitou a autorização para a sua realização, além da utilização formal do nome da instituição no relatório final da investigação. Os pesquisadores se comprometeram, nesse ofício, a honrar os princípios científicos, éticos e legais que regem a pesquisa com bancos de dados, cujas informações são agregadas, sem possibilidade de identificação individual, preconizados na Resolução $\mathrm{n}^{\circ}$ 541/2016 do Conselho Nacional de Saúde.

Após a aprovação do Secretário Estadual de Saúde do Rio Grande do Norte deu início a coleta dos dados.

O tratamento dos dados foi realizado por meio do programa Microsoft Office Excel $\circledast$ e submetido ao programa SPSS 25.0 
Tabela 1. Caracterização sociodemográfica das vítimas de maus-tratos, abandono e negligência no Estado do Rio Grande do Norte. Natal/RN, Brasil, 2019.

\section{Categorias}

n

Sexo

\section{Feminino}

Masculino

Faixa etária

Criança (0 a 9 anos)

Adolescente (10 a 19 anos)

Jovem (20 a 24 anos)

Pessoa adulta ( 25 a 59 anos)

Pessoa idosa (60 anos ou mais)

Orientação sexual

\begin{tabular}{lcc|}
\hline Heterossexual & 69 & 15,8 \\
\hline Homossexual (gay/lésbica) & 3 & 0,7 \\
\hline Não se aplica* & 315 & 72,2 \\
\hline Ignorado & 49 & 11,2 \\
\hline Identidade de gênero & & \\
\hline Mulher Transexual & 2 & 0,5 \\
\hline Não se aplica* & 376 & 86,2 \\
\hline Ignorado & 58 & 13,3 \\
\hline Raça/Cor & & \\
\hline Parda & 162 & 37,2 \\
\hline Branca & 66 & 15,1 \\
\hline Preta & 20 & 4,6 \\
\hline Amarela & 1 & 0,2 \\
\hline Indígena & 1 & 0,2 \\
\hline Ignorado & 182 & 41,7 \\
\hline Escolaridade & & \\
\hline Analfabeto & 8 & 1,8 \\
\hline Ensino fundamental incompleto/completo & 84 & 19,2 \\
\hline Ensino médio incompleto/completo & 2 & 0,5 \\
\hline Ensino superior incompleta/completo & 2 & 0,4 \\
\hline Ausente & 19 & 4,4 \\
\hline Não se aplica* & 374 & 49,1 \\
\hline Ignorado & 214 & 24,5 \\
\hline Grávida & 107 & \\
\hline Não & & 9,6 \\
\hline Sim & 42 & 0,7 \\
\hline Não se aplica* & 3,9 \\
\hline Ignorado & & \\
\hline Zona & & \\
\hline
\end{tabular}

para realização de limpeza e verificação de consistência dos dados notificados, bem como a análise descritiva. Após o tratamento do banco de dados, foi realizada uma análise descritiva através das medidas do centro de distribuição da variabilidade ou variabilidade e dispersão para variável quantitativa e a utilização de valores absolutos e porcentagens das variáveis qualitativas. A construção de tabelas ou gráficos foi realizado no utilizando Microsoft Office Excel® 365.

\section{RESULTADOS}

No período de 2015 a 2018 foram notificados 12.665 casos de violência no Estado do Rio Grande do Norte, sendo $436(3,4 \%)$ casos de maus-tratos, negligência ou abandono. $\mathrm{O}$ maior percentual de notificações ocorreu no ano de 2017 (142), seguido de 2018 (130).

No que se refere a caracterização das vítimas que sofreram maus-tratos, negligência ou abandono, através da análise da tabela 1, observa-se que a maior parte era do sexo feminino $(59,6 \%)$, na faixa etária de 0 a 9 anos de idade (56,4\%), heterossexual $(15,8 \%)$, sem identificação da identidade de gênero $(86,2 \%)$, da raça/cor parda $(37,2 \%)$, com ensino fundamentalincompleto/completo $(19,2 \%)$, não estava gestante $(85,8 \%)$, da zona urbana $(79,1 \%)$, das que tinham idade para ter um relacionamento, a maior parte $(11,7 \%)$ era solteiro.

Foram notificadas 37 (8,5\%) pessoas que possuíam algum tipo de deficiência/ transtorno, no entanto, existia percentual significante $(23,0 \%)$ de dados registrados como ignorados ou ausentes. Dos que possuíam algum tipo transtorno, a maioria foi notificada como deficiência física (35,0\%).

Com relação aos dados de ocorrência da violência relacionada à maus-tratos, abandono e negligência, observa-se na Tabela 2, que a maioria ocorreu na zona urbana $(76,6 \%)$, sofreu violência na própria residência $(82,8 \%)$ e já havia ocorrido outras vezes $(44,5 \%)$

Quanto ao tipo de violência sofrido, mostrando que a violência psicológica/moral $(26,6 \%)$ foi predominante, seguida da 
Urbana 345

\begin{tabular}{lcc|} 
Rural & 81 & 18,6 \\
\hline Periurbana & 1 & 0,2 \\
\hline Ausente & 7 & 1,6 \\
\hline Ignorado & 2 & 0,5 \\
\hline Situação conjugal / Estado civil & & \\
\hline Solteiro & 51 & 11,7 \\
\hline Casado/união consensual & 30 & 6,9 \\
\hline Viúvo & 11 & 2,5 \\
\hline Separado & 4 & 0,9 \\
\hline Não se aplica* & 300 & 68,8 \\
\hline Ignorado & 28 & 6,4 \\
\hline Ausente & 12 & 2,8
\end{tabular}

Fonte: SVS/MS *dado registrado com pessoa com pouca idade ou deficiência

Tabela 2. Pessoas vítimas de maus-tratos, abandono e negligência no Estado do Rio Grande do Norte segundo os dados da ocorrência do evento. Natal/RN, Brasil, 2019.

\section{Categoria}

n

Zona de Ocorrência

\begin{tabular}{lcc|}
\hline Urbana & 334 & 76,6 \\
\hline Rural & 71 & 16,3 \\
\hline Periurbana & 1 & 0,2 \\
\hline Ignorada & 2 & 0,5 \\
\hline Ausente & 28 & 6,4 \\
\hline Local de Ocorrência & & \\
\hline Residência & 361 & 82,8 \\
\hline Habitação coletiva & 2 & 0,5 \\
\hline Escola & 3 & 0,7 \\
\hline Local de prática esportiva & 1 & 0,2 \\
\hline Via pública & 11 & 2,5 \\
\hline Outros & 32 & 7,3 \\
\hline Ignorado & 25 & 5,7 \\
\hline Ausente & 1 & 0,2 \\
\hline
\end{tabular}

Ocorreu outras vezes?

\begin{tabular}{lcc} 
Sim & 194 & 44,5 \\
Não & 62 & 14,2 \\
\hline Ignorado & 176 & 40,4 \\
Ausente & 4 & 0,9
\end{tabular}

Fonte: SVS/MS

Tabela 3. Caracterização dos agressores de maus-tratos, abandono e negligência no Estado do Rio Grande do Norte. Natal/RN, Brasil, 2019.

$$
\text { Categoria }
$$$$
\text { n }
$$

Porcentagem

Porcentagem

res. Violências e agressões praticadas no âmbito familiar podem prejudicar o bem-estar, a integridade física e ou psicológica, a liberdade e o direito ao pleno desenvolvimento das pessoas, em especial, das crianças. ${ }^{6,7}$

Com relação as características das pessoas que sofreram maus-tratos, negligência ou abandono, os resultados do presente estudo se assemelham a outras pesquisas realizadas sobre a temática, nas quais mostram que a maior parte foi o sexo feminino e menores de nove anos de idade. Este fato é justificado por situações de autoritarismo e dominação, replicando situações de opressão em populações mais vulneráveis. Autores têm atribuído aspectos culturais que reforçam uma visão masculina da sociedade, aplicando atitudes violentas de forma mais intensa contra meninas e crianças mais jovens. ${ }^{7}$

Com relação ao local de ocorrência dos maus-tratos, observa-se que o domicílio foi o mais frequente, dados confirmados por outras pesquisas. Pelo fato deste tipo de violência ter ocorrido mais em crianças e estas permanecerem mais tempo de suas vidas no domicílio, a ocorrência de violências torna-se mais frequente neste local. A residência deveria representar um ambiente de proteção e de cuidado, mas torna-se o local de agressão e de vitimização de determinadas crianças. ${ }^{7.8}$

O presente estudo mostrou resultados 
Feminino 188

\begin{tabular}{lcc|} 
Ambos os sexos & 128 & 29,4 \\
\hline Masculino & 87 & 20,0 \\
\hline Ignorado/ausentes & 33 & 7,5 \\
\hline Ingestão de bebida alcoólica & & \\
\hline Não & 164 & 37,6 \\
\hline Sim & 97 & 22,2 \\
\hline Ignorado/ausente & 175 & 40,1 \\
\hline
\end{tabular}

Ciclo de vida do provável autor da violência

\begin{tabular}{lcc|} 
Criança (0 a 9 anos) & 6 & 1,4 \\
\hline Adolescente (10 a 19 anos) & 28 & 6,4 \\
\hline Jovem (20 a 24 anos) & 22 & 5,0 \\
\hline Pessoa adulta (25 a 59 anos) & 290 & 66,5 \\
\hline Pessoa idosa (60 anos ou mais) & 11 & 2,5 \\
\hline
\end{tabular}

Ignorado

79

18,1

Violência motivada por:

Conflito geracional

87

Sexismo

20,0

Situação de rua

20

Deficiência

6

6

4,6

\section{Outros}

4

49

Não se aplica

180

90

80

Ignorado

1,4

Fonte: SVS/MS

graves e preocupantes, pois além da vulnerabilidade das vítimas relacionada à idade, uma vez que $56,4 \%$ tinham menos de nove anos, ainda $8,5 \%$ delas apresentavam deficiências de ordem física, mental e visual ou transtornos. É importante destacar que o reconhecimento dos sinais de maus-tratos entre crianças e adolescentes com defici- ência intelectual pode ser dificultado pela incapacidade que eles têm de verbalizar que estão sendo maltratados, ou por não compreenderem que estão sendo agredidos. Naqueles com deficiência física, a dificuldade em falar sobre a violência sofrida pode estar associada ao fato de quem agride é o mesmo que cuida, havendo elos afetivos, além do receio do abandono após a identificação de maus-tratos. ${ }^{9}$

\section{CONCLUSÃO}

O presente estudo descreveu a situação epidemiológica de pessoas que foram vítimas de maus-tratos, negligência ou abandono em um estado do nordeste brasileiro, chagando as seguintes conclusões:

Quanto as características das pessoas que foram vítimas de maus-tratos, negligência ou abandono, observou-se que a maior parte era do sexo feminino, na faixa etária de 0 a 9 anos de idade, heterossexual, sem identificação da identidade de gênero, da raça/cor parda, com ensino fundamental incompleto/completo, não estava gestante, da zona urbana. Das que tinham idade para ter um relacionamento, a maior parte era solteiro.

Quanto ao perfil das pessoas que cometeram maus-tratos, negligência ou abandono, observou-se maioria era do sexo feminino, não havia ingerido bebida alcóolica, era adulta, na faixa de 25 a 59 anos e a violência foi motivada por conflito geracional.

Desse modo, a pesquisa evidenciou a necessidade de políticas públicas que proteja as crianças e as pessoas que estão em situação de vulnerabilidade. As leis existentes atualmente demonstram-se ineficazes para promoção e proteção do menor e também para os portadores de algum tipo de deficiência ou transtorno, ou seja, necessidades especiais trazendo à tona o cenário de abandono, falta de fiscalização e intervenções preventivas.

\section{Referências}

1. Minayo MCS. Violência e saúde. Rio de Janeiro: FIOCRUZ; 2010.

2. Silva LEL, Oliveira MLC. Violência contra a mulher: revisão sistemática da produção científica nacional no período de 2009 a 2013. Ciênc Saúde Coletiva. 2015; 20(11): 3523-3532.

3. Brasil. Ministério da Saúde. Viva: instrutivo de notificação de violência interpessoal e autoprovocada. Brasília (DF): Ministério da Saúde; 2016.

4. Brasil. Ministério da Saúde. Mapa da violência 2014: homicídios e juventude no Brasil: atualização de 15 a 29 anos. Brasília (DF): Ministério da Saúde; 2014. 5. Fonseca DH, Ribeiro CG, Leal NSB. Violência doméstica contra a mulher: realidades e representações sociais. Psic e Soc. 2012; 24(2): 307-314.

6. Machado JC, Rodrigues VP, Vilela ABA, Simões AV, Morais RLGL, Rocha EN.
Violência intrafamiliar e as estratégias de atuação da equipe de Saúde da Família. Saude Soc. 2014; 23(3): 828-840.

7. Malta DC, Antunes JT, Prado RR, Assunção AA, Freitas MI. Fatores associados aos episódios de agressão familiar entre adolescentes, resultados da Pesquisa Nacional de Saúde do Escolar (PeNSE). Ciênc Saúde Coletiva. 2019; 24(4): 1287-1298.

8. Abranches $C D$, Assis SG. A (in)visibilidade da violência psicológica na infância e adolescência no contexto familiar. Cad Saúde Pública. 2011; 27(5): 843-854.

9. Barros CRS, Schraiber LB. Violência por parceiro íntimo no relato de mulheres e de homens usuários de unidades básicas. Ver Saúde Pública. 2017; 51(7). 